NOTICE WARNING CONCERNING COPYRIGHT RESTRICTIONS:

The copyright law of the United States (title 17, U.S. Code) governs the making of photocopies or other reproductions of copyrighted material. Any copying of this document without permission of its author may be prohibited by law. 


\title{
Fast Randomized Consensus Using Shared Memory
}

\author{
James Aspnes, Maurice Herlihy \\ December 20, 1988 \\ CMU-CS-88-205 2
School of Computer Science
Carnegie Mellon University
Pittsburgh, PA 15213

\begin{abstract}
We give a new randomized algorithm for achieving consensus among asynchronous processes that communicate by reading and writing shared registers. The fastest previously known algorithm, due to Abrahamson, has expected running time $2^{O\left(n^{2}\right)}$. Our algorithm is polynomial, requiring an expected $O\left(n^{4}\right)$ operations. Applications of this algorithm include the elimination of critical sections from concurrent data structures and the construction of asymptotically unbiased shared coins.
\end{abstract}

This research was partially sponsored by the Office of Naval Research (DOD), under Contract N00014-88-K-0641. The work of J. Aspnes was supported by a National Science Foundation Graduate Fellowship.

The views and conclusions contained in this document are those of the authors and should not be interpreted as representing the official policies, either expressed or implied, of the Defense Advanced Research Projects Agency, the National Science Foundation or the U.S. government. 


\section{Introduction}

A consensus protocol is a set of $n$ processors, running asynchronously, that communicate by applying operations to a shared object. The object may be a message channel, an array of read/write registers, or something more complex. Each process starts with an input value, either 0 or 1 , and runs until it chooses a decision value and halts. A consensus protocol is correct if it is consistent: no two processes choose different decision values, non-trivial: the decision value was some process's input value, and wait-free: each process decides after a finite number of steps.

Consensus protocols are interesting because they are fundamental to synchronization without mutual exclusion. The traditional approach to coordinating concurrent access to shared data objects is to rely on critical sections: only one process at a time is allowed to operate on the object. Nevertheless, critical sections are poorly suited for asynchronous, fault-tolerant systems: if a faulty process is halted or delayed in a critical section, non-faulty processes will also be halted or delayed. By contrash, an implementation of a concurrent data object is wait-free if it guarantees that any process will complete any operation in a finite number of steps, independent of other processes' halting failures or variations in speed. If there exists a consensus protocol for an object $X$, then one can use $X$ to construct a wait-free implementation of any concurrent data object whose operations are total [21].

If the shared object $\mathrm{X}$ is an array of registers providing read and write operations, then consensus is known to be impossible $[2,12,21,26]$. If $\mathrm{X}$ is an array of registers providing test-and-set or fetch-and-add operations, then consensus is possible $h$ 'reen two processes, but not among three [21]. Nevertheless, in both cases, consensus among an arbitrary number of processes can still be achieved probabilisticaily. This paper presents two new randomized consensus protocols, one in which processes communicate by reading and writing shared registers, and one in which they communicate by applying fetch-and-add operations. The protocols are consistent, non-trivial, and they guarantee that each process decides after a finite expected number of steps. The only previously known read/write protocol, due to Abrahamson [1], requires an expected $2^{O\left(n^{2}\right)}$ operations. Ours is significantly faster, requiring an expected $O\left(n^{2}\right)$ writes and $O\left(n^{4}\right)$ reads. The fetch-and-add protocol requires an expected $O\left(n^{2}\right)$ fetch-and-add operations.

The basic idea behind our protocols is quite simple. We first describe a simple protocol that has exponential expected running time if an adversary scheduler runs the processes in lockstep. Each process flips an unbiased coin at each round, and the protocol halts when all $n$ processes simultaneously flip the "right" value. The probability of terminating at any particular round is $1 / 2^{n}$, so the expected number of rounds until termination is $2^{n}$. A naive approach to speeding up the protocol is to replace the $n$ independent coin flips with a single unbiased coin shared by the processes. Unfortunately, implementing an unbiased shared coin is provably impossible in an asynchronous system (see Section 8 below), so it would appear that no progress has been made. The key insight, however, is similar to one proposed by Chor, Merrith, and Shmoys [10]: it suffices to ensure that processes are sufficiently likely to flip the same value, and that an adversary scheduler has a sufficiently weak influence over which value is chosen. The hear of our consensus protocol is a weak shared coin protocol that guarantees: (1) processes are likely to observe the same outcome, (2) an adversary scheduler has only a weak influence over that outcome, and (3) the protocol has expected running time polynomial in the number of processes.

Consensus is often viewed as a problem in game theory. One side, the processes, tries to achieve agreement against an adversary scheduler. The processes apply read and write operations to the shared registers, and the adversary chooses when the operations actually occur. The adversary is extremely powerful: it has complete information about the processes' protocols, their internal states, and the state of the shared memory. The adversary is not restricted to polynomial resources, thus it cannot be outwitted by encryption schemes. The adversary cannot, however, predict future coin flips. Against such a powerful adversary, it may seem surprising that consensus can be achieved by a simple protocol in polynomial expected time. 


\section{Related Work}

Fischer, Lynch, and Paterson [19] show that there is no consensus protocol for two processes that communicate by asynchronous messages. Dolev, Dwork, and Stockmeyer [14] and Dwork, Lynch, and Stockmeyer [16] give a comprehensive analysis of the circumstances under which consensus can be achieved by message-passing. Randomized protocois can achieve consensus when deterministic protocols cannoL Ben-Or [3] proposes a randomized consensus protocol with exponential expected nunning time that wlerates up to $n / 5$ failures, where $n$ is the number of processes. A consensus protocol due to Bracha and Toueg si relies on probabilistic properties of the message-passing system.

Loui and Abu-Amara [26] give several consensus protocols and impossibility results for processes that communicate through shared registers with various read-modify-write ("test-and-set") operations. Chor, Israeli and $\mathrm{Li}$ [12] give two randomized consensus protocols for shared read/write registers, one for two processes, and one for three processes. 1 Their protocols, however, are correct only if one assumes that a process can write a register and change state in a single atomic transition. They do not work in the model used in this paper, where updating a register and changing process state are considered to be distinct transitions. Abrahamson [1] gives consensus protocols for both the "strong" model used by Chor, Israeli, and Li, and the more demanding "weak" model used here. As mentioned above, Abrahamson's consensus protocol for the weak model has exponential expected running time.

A number of protocols have been proposed for implementing shared coins in message-passing systems subject to byzantine or halting failures. Some constructions are direct $[4,8,15]$, and others arise as parts of protocols for consensus [10], transaction commitment [13], or byzantine agreement $[7,10,17,32]$. The models underlying these protocols differ from ours by assuming that private channels or encryption can be used to prevent the adversary from observing certain messages and processes' internal states. Chor and Coan [11] give a randomized byzantine agreement protocol that does not assume private communication, but restricts when the adversary may exploit knowledge of the processes' states.

\section{Model}

\subsection{V/O Automata}

Formally, we model processes and registers as $1 / O$ automata $[27,28]$. An $I / O$ automaton is a non-deterministic automaton $\mathbf{A}$ with the following components:

- states $(A)$ is a finite or infinite set of states, including a distinguished set of starting states.

- in(A) is a set of input events.

- $\operatorname{out}(A)$ is a set of ouput events,

- $\operatorname{steps}(A)$ is a transition relation given by a set of triples $\left(s^{\prime}, e, s\right)$, where $s$ and $s^{\prime}$ are states and $e$ is an event Such a triple is called a step, and it means that an automaton in state $s$ can undergo a transition to state $s$, and that transition is associated with the event $e$.

If $\left(s^{\prime}, e, s\right)$ is a step, we say that $e$ is enabled in $s^{\prime}$. I/O automata must satisfy the additional condition that inputs cannot be disabled: for each input event $e$ and each state $s^{\prime}$, there exist a state $s$ and a step $\left(s^{\prime}, e, s\right)$.

An execution of an automaton $A$ is a finite sequence $s_{0}, e_{1}, s_{1}, \ldots, e_{n}, s_{n}$ or infinite sequence $s_{0}, e_{1}, s_{l}, \ldots$ of alternating states and events such that $s_{0}$ is a starting state and each $\left(s_{i}, e_{i+1}, s_{i+1}\right)$ is a step of A. A history of an

'The three-process prococol published in [12] has a bug: the termination condition must be strengthened so ensure consistency. 
automaton is the subsequence of events occurring in one of its executions.

A new L/O automaton can be constructed by compc.ing a set of L/O automata with disjoint output events. A state of the composed automaton $S$ is a tuple of component states, and a starting state is a tuple of component starting states. The set of events of $S$, events( $S$ ), is the union of the components' sets of events, and the set of output events of $S$, out (S), is the union of the components' sets of output events. The sets of input events of $S$, in $(S)$, is events $(S)$ $o u t(S)$, all the events of $S$ that are not output events for some component. A triple $\left(s^{\prime}, e, s\right)$ is in steps $(S)$ if and only if, for all component automata $A$, one of the following holds: (1) $e$ is an event of $A$, and the projection of the step onto $\mathrm{A}$ is a step of $\mathrm{A}$, or (2) $e$ is not an event of $\mathrm{A}$, and $\mathrm{A}$ 's state components are identical in $s^{\prime}$ and $s$. If $\mathrm{H}$ is a history of a composite automaton and $\mathrm{A}$ an automaton, $\mathrm{H}$ I A denotes the subhistory of $\mathrm{H}$ consisting of events of $\mathrm{A}$.

\subsection{Processes, Coins, and Registers}

A process $P$ is an $I / O$ automaton with output events WRITE( $P, v, R), \operatorname{READ}(P, R)$, and $D E C I D E(P, v)$; input event RETURN $(P, v, R)$; and internal event COIN-FLIP $(P, x)$, where $v$ is a value, $R$ a register, and $x$ (the value of the coin-flip) an element of the set $(0,1)$. The two COIN-FLIP events of a process represent possible results of a random decision made within the process; if either is enabled in a particular state, the other must also be enabled. READ and WRITE events are called invocations, and RETURN events are called a responses. An invocation and response match if their process and object names agree. An operation is a pair consisting of an invocation and the next matching response. An invocation with no matching response is pending. To capture the notion that a process represents a single thread of control, we say that a process history is well-formed if every response is immediately preceded by a matching invocation.

A register $R$ is an $\mathrm{L} / \mathrm{O}$ automaton with input events WRITE(P, $v, R)$ and $R E A D(P, R)$, and the output event $\operatorname{RETURN}(P, v, R)$, where $P$ is a process and $v$ a value.

\subsection{Protocols}

Let $P=\left\{P_{1}, \ldots, P_{n}\right\}$ be a set of processes and $R=\left\{R_{1}, \ldots, R_{m}\right\}$ a set of registers. The protocol $\angle P, R>$ is the $I / O$ automaton composed by identifying invocations and responses for processes $P_{1}, \ldots, P_{n}$ and registers $R_{1}, \ldots, R_{m}$. A protocol history is well-formed if each $H I P_{i}$ is well-formed, and a protocol is well-formed if each of its histories is well-formed. We restrict our attention to well-formed protocols.

A protocol history $H$ is sequential if, for each register $R_{j}, H \mid R_{j}$ begins with an invocation and alternates matching invocations and responses. If we restrict our attention to sequential histories, then the behavior of a register can be specified in a particularly simple way: the value read is the last value written. Each history $\mathrm{H}$ induces a partial "real-time" order $<_{\mathrm{H}}$ on its operations: $o p_{0}<_{\mathrm{H}} o p_{I}$ if the response for $o p_{0}$ precedes the invocation for $O p_{l}$. Operations unrelated by $<_{\mathrm{H}}$ are said to be concurrent. If $\mathrm{H}$ is sequential, $<_{\mathrm{H}}$ is a total order.

A protocol $\left[P_{1}, \ldots, P_{n} ; R_{1}, \ldots, R_{m}\right\}$ is linearizable [20] if, for each history $H$, there exists a sequential history $S$ such that:

- For all $\mathrm{P}_{\mathrm{i}}, \mathrm{H}\left|\mathrm{P}_{\mathrm{i}}=\mathrm{S}\right| \mathrm{P}_{\mathrm{i}}$.

$\bullet<_{\mathrm{H}} \subseteq<_{\mathrm{S}}$

In other words, the history "appears" sequential to each individual process, and this apparent sequentiat interleaving respects the real-time precedence ordering of operations. Each read $\alpha$ write appears to take effect instantaneously at some point between its invocation and its response. We restrict our attention to linearizable protocols. 


\subsection{Randomization}

The random non-determinism involved in the choice between COIN-FLIP $(P, 0)$ and COLN-FLIP $(P, 1)$ has a different nature from the "ordinary" non-determinism in the protocol. It is possible to make the distinction formally by placing the other non-deterministic choices under the control of an adversary, a function A which maps each of the protocol's finite executions $s_{0}, e_{1}, s_{1}, \ldots, e_{n}, s_{n}$ to a set of events enabled in $s_{n}$, such that for all finite executions $\xi, A(\xi)$ consists either of a single non-coin-flip event or a pair of coin-flip events representing the two possible outcomes of a coin-flip at some process. The intent is that the adversary controls which executions are possible; more formally, we say that an adversary $A$ permits a (possibly infinite) execution $\xi=s_{0}, e_{1}, s_{1}, \ldots$, if, for every event $\mathrm{e}_{\mathrm{i}}$ in $\xi, \mathrm{e}_{\mathrm{i}}$ is an element of $\mathrm{A}\left(s_{0}, e_{1}, s_{1}, \ldots, \mathrm{s}_{\mathrm{i}-1}\right)$. We write $\Xi_{\mathrm{A}}$ for the set of executions that $\mathrm{A}$ permits, and $\Xi_{A, S}$ for the subset of $\Xi_{A}$ consisting of executions which have $s$ as their initial state.

Let $c(\xi)$ be the sequence of coin-flip values in $\xi$. It is a straightforward consequence of the constraints on the domain of an adversary function $A$ that, for each countable sequence $C$ of 0 's and 1 's, there exists exactly one $\xi_{A, S}(C)$ in $\Xi_{A, S}$ such that $c\left(\xi_{A, S}(C)\right)$ is a prefix of $C$. We can think of $\xi_{A, S}$ as a measurable function from the sequence space $\Omega$ on the set $\{0,1\}$ to the sequence space $\Xi$ of protocol executions. ${ }^{2}$ We can thus use $\xi_{A, s}$ to define a probability measure on $\Xi$ by transforming the probability measure on $\Omega$ as follows:

$$
P_{A, S}(X)=P\left(\left\{C \mid \xi_{A, S}(C) \in X\right]\right)
$$

(where the probability on the left is defined only when $X \subseteq \Xi$ is measurable $\Xi$ ). An immediate consequence of the definition is that $P_{A, 5}(X)=0$ for any $\mathrm{X}$ which is disjoint from $\Xi_{\mathrm{A}, \mathrm{s}}$

\subsection{Consensus Protocols}

A consensus protocol is a protocol whose processes each have two initial states, corresponding to input values of 0 or 1 , respectively, and whose histories all satisfy the following conditions:

1. Consistency. Every DECIDE event in the history has the same value, which must be an input value for at least one of the processes.

2. Termination A DECIDE event for $P$ must be the last output event of $P$.

3. Non-triviality. If $s$ is an input state in which some processes start with different values, there exist adversaries $A, B$ such that $P_{A, s}(\{\xi \mid \xi$ contains $D E C I D E(P, 0)$ for some $P\})$ and $P_{B, s}(\{\xi \mid \xi$ contains $\operatorname{DECIDE}(P, 1)$ for some $\mathrm{P}\})$ are both non-zero.

The first condition guarantees that the protocol actually achieves consensus. The second condition is not critical to describing a consensus protocol, but is necessary for identifying when a protocol is finished. The third condition excludes protocols which achieve consensus trivially by fixing the outcome in advance.

The running time $\mathrm{r}(\mathrm{H})$ of a protocol history $\mathrm{H}$ is the length of the shortest prefix of $\mathrm{H}$ which contains a DECIDE event for every process in the protocol. The worst-case expected running time of a protocol is given by

$$
\max _{A, S} \sum_{i=0}^{\infty} i \cdot P_{A, S}(\{\xi \mid r(\xi)=i\})
$$

which is simply the expected nunning time of the protocol against the worst possible adversary. If, for some adversary $A$ and initial state $s$, the sum in the above expression does not converge, we say that the protocol has an infinite worst-case expected running time.

\footnotetext{
${ }^{2}$ For a detriled discussion of sequence spaces, see [22]. We will assume throughout that each branch in $\Omega$ has weight $1 / 2$, i.e. that our coins are fair.
} 


\section{An Exponential Consensus Protocol}

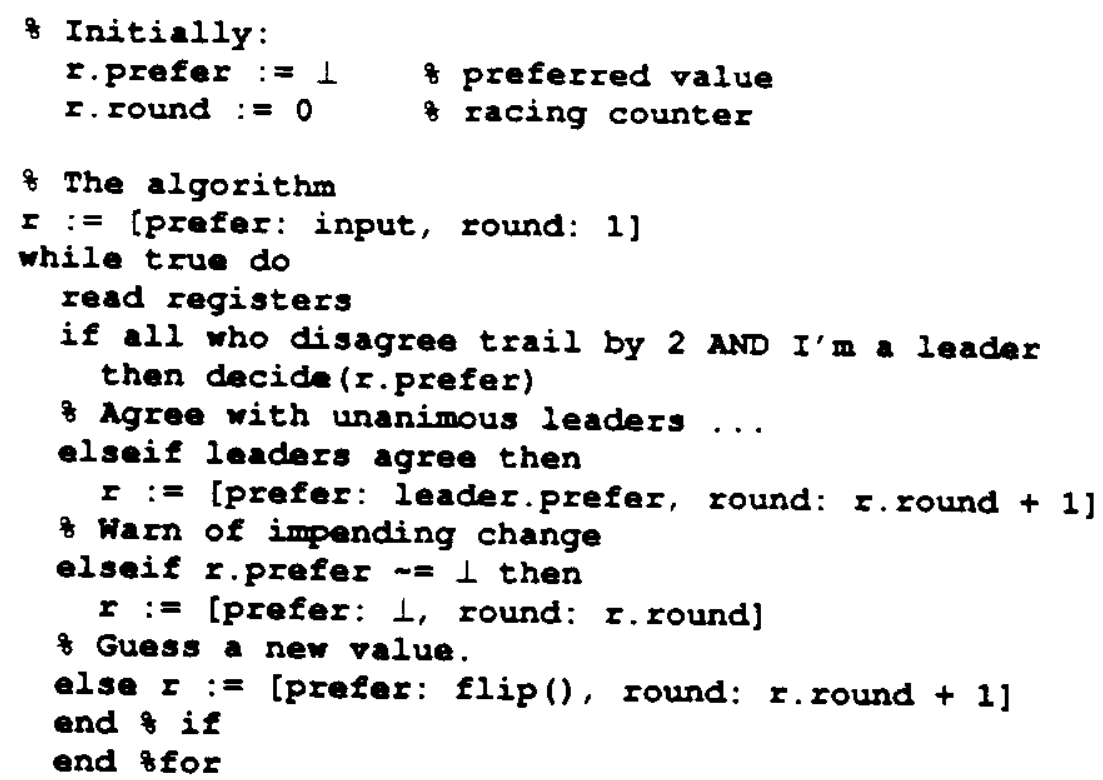

Figure 41: An Exponential Consensus Protocol

Each process $P$ has a register with two fields:

- prefer, if distinct from $\perp$, is the value $P$ would choose if it were to complete the protocol executing in isolation,

- and round is a counter that keeps track of the number of rounds $P$ has executed so far.

A process $Q$ agrees with $P$ if (1) both prefer fields are equal, and (2) neither is $\perp$. A process is a leader if its rounds field is greater than or equal to any other process's rounds field.

The protocol, shown in Figure 4-1, works as follows. Initially, P's register is initialized so that round is 0 and prefer is $L$. Process $P$ starts by setting round to 1 , and prefer to its input value. $P$ then enters the main loop of the protocol. It reads all processes' registers. The protocol terminates if $P$ is a leader, and if all processes whose round fields trail P's by less than two agree with $P$. Otherwise, if the leaders agree, $P$ updates its register to agree with the leaders, increments its round counter, and resumes the loop. Otherwise, if its prefer field is not $1, P$ "warns" the other processes that it may change its preference by setting prefer to $\perp$ before resuming the loop. If prefer is already $\perp$, then $P$ chooses a new preference by an unbiased coin flip, increments round, and resumes the loop.

Although the rounds field is potentially unbounded, larger values are reached with lower probabilities, thus the likelihood of overflow can be made arbitrarily small.

\subsection{Consistency}

Let $\mathrm{H}$ be a history (sequence of reads, writes, and flips) permitted by a particular adversary. For brevity, we say that process $P$ prefers $v \neq \perp$ at round $r$ if $P$ writes [prefer: $v$, round: $r$ ] at some step in $H$, and that $P$ is busy at round $r$ if it writes [prefer: 1 , round: $r$ ]. The first process to prefer $v$ at round $r$ is the one whose write occurred earliest in $\mathrm{H}$. We use $v$ and $v^{\prime}$ to stand for the two distinct decision values.

Lemma 1: If $\mathrm{P}$ prefers $v$ at round $r$ and $v^{\prime}$ at round $r+1$, then some $\mathrm{Q} \neq \mathrm{P}$ prefers $v^{\prime}$ at round $r^{\prime} \geq r$, and Q's write precedes $P$ 's write of $v^{\prime}$.

Proof: P can change preference from $v$ to $v^{\prime}$ in one of two ways: if it observes that all leaders agree on 
$v^{\prime}$, or if it observes that one leader prefers $v$ and another $v^{\prime}$. In either case, some other $Q$ prefers $v^{\prime}$ at round $r^{\prime} \geq r$, and since $P$ read that value, Q's write of $v^{\prime}$ must precede $\mathrm{P}^{\prime} s$.

Lemma 2: If every process that completes round $r$ in $\mathrm{H}$ prefers $v$ at that round, then no process prefers a distinct value at any higher round.

Proof: Suppose not. Let $\mathrm{P}$ be the first process in $\mathrm{H}$ to prefer $v^{\prime}$ at round $r^{\prime}>r$. Lemma 1 implies that some Q prefers $v^{\prime}$ at round $r^{\prime \prime} \geq r$. Since it is given that all processes that completed round $r$ prefer $v$, it follows that $r^{\prime \prime}>r$, contradicing the hypothesis that $\mathrm{P}$ is the first process to switch its preference after round $r$.

By similar reasoning:

Lemma 3: If every process that completes round $r$ in $H$ prefers $v$ at that round, then no process is busy at any higher round.

Lemma 4: If every process that completes round $r$ in $\mathrm{H}$ prefers $v$ at that round, then every process that completes round $r+1$ decides $v$ before completing round $r+2$.

Proof: By contradiction. Any process that decides after round $r$ must decide $v$, since Lemma 2 implies it must prefer that value. Let $Q$ be the first process to read at the start of round $r+2$. Since no other process has started that round, $Q$ is a leader. If $Q$ fails to decide, or it disagrees with another leader $P$ at round $r+1$. The first case contradicts our assumption that $Q$ is the first process to finish round $r+1$. In the second case, since $Q$ prefers $v$ at round $r$, either $Q$ prefers $v^{\prime} \neq v$ at round $r+1$, contradicting Lemma 1 , or $Q$ is busy at round $r+1$, contradicting Lemma 3 .

Lemma 5: If $\mathrm{P}$ decides $v$ at round $r$ in $\mathrm{H}$, then no other process prefers $v^{\prime}$ at round $r$.

Proof: Suppose not. Let Q be the first process to prefer $v^{\prime}$ at round $r$ in H. Since P decided $v$, it must have observed one of two situations when it last read Q's register. (1) Q prefers $v$ at round $r-1$, or (2) Q's round was $r^{\prime}<r-1$.

In the first case, we have the following sequence of steps. $P$ writes [prefer: $\nabla$, round: $r$ ], and later reads [prefer: $\nabla$, round: $r-1$ ] from $Q$ 's register. Before $Q$ can switch its preference, however, it must set its prefer field to $\perp$ and reread the registers. $Q$ now observes that $P$ prefers $v$ at round $r$. By hypothesis, no other process prefers $v^{\prime}$ at round $r$, hence by Lemma $2 \mathrm{Q}$ observes that the leaders agree on $v$. Q must reset its register to agree with the leaders, a contradiction.

The second case is similar. P sets its register to [prefer: $\nabla$, round: $r$ ]. P later reads Q's register, which has round $r^{\prime}<r-1$. Q advances its round to $r^{\prime}+1<r$ and rereads $P$ 's register, observing that $\mathrm{P}$ prefers $v$ at round $r$. By hypothesis, no other process prefers $v^{\prime}$ at round $r$, hence by Lemma $2 \mathrm{Q}$ observes that the leaders agree on $v$, and $Q$ resets its register to agree with the leaders, a contradiction.

Theorem 6: This consensus protocol is consistent.

Proof: If any process decides on $v$ at round $r$, then all processes will prefer $v$ at round $r$ (Lemma 5), and hence all processes will eventually decide $v$ (Lemma 4).

This protocol can be extended to allow decision values from an arbitrary domain, not just $\{0,1\}$. Before joining the protocol, each process writes its initial value to a public register. Instead of flipping a coin to change preference, a process randomly adopts a leader's preference.

\subsection{Running Time}

The adversary scheduler can force the protocol to nu forever if it can ensure that at each round, two processes have different preferences.

Let $\mathrm{V}$ and $\mathrm{V}^{\prime}$ be the sets of processes that respectively prefer $v$ and $v^{\prime}$ at round $r$.

Lemma 7: The set of processes that randomly chose their round $r$ preferences encompasses at least one of $V$ and $V '$.

Proof: We show that if $P$ and $Q$ belong to $V$ and $V$ ', then at least one of the two must have chosen its 
preference by a coin flip.

Let $P$ be the furst process to write a preference, say $v$, at round $r$. If $P$ did not choose $v$ by flipping a coin, then $P$ must have observed that the leaders prefer $v$. Since $P$ is the first process to enter round $r$, it must have observed that all processes at round $r-1$ prefer $v$. Let $Q$ be the first process to prefer $v^{\prime}$ at round $r$. If $Q$ did not flip a coin, then it must have observed that the leaders prefer $v^{\prime}$. Since all processes at rounds $r$ and higher prefer $v, Q$ must have observed that all processes at round $r-1$ prefer $v^{\prime}$. Each process, however, writes out its preference for round $r-1$ before reading the other's register, thus at least one of the two must have observed a disagreement before entering round $r$, and that process must have performed a coin flip.

Theorem 8: The consensus protocol has a worst-case expected running time of $O\left(2^{n}\right)$ rounds.

Proof: Consider the set of coin flips associated with each round. If the set is empty, Lemma 7 implies that the processes have identical preferences, and Lemma 4 implies that protocol is about to terminate. If the set is non-empty, then Lemma 7 implies that all the processes that chose a preference for round $r$ without flipping chose the same preference, say $v$. If all the processes that choose randomly also choose $v$, then, by Lemma 4 , the protocol will terminate. Since there are at most $n$ coin flips at each round, and since exactly one combination of flips can terminate the protocol, this protocol has expected running time no worse than $2^{n}$ rounds. This bound is easily seen to be tight. The adversary can un the processes in lockstep, so that all $n$ processes observe disagreement at each round, and all flip to choose a preference for the next round.

Corollary 9: The consensus protocol has a worst-case expected running time of $\mathrm{O}\left(2^{n}\right)$ steps.

Proof: In each round, each process performs at most $n$ READ invocations, one COIN.FLIP, and one WRITE invocation, for a total of $2 n+3$ steps. Thus the total number of steps taken per round by all processors is at most $n^{2}$, giving a maximum total running time of $O\left(n^{2} 2^{n}\right)=O\left(2^{n}\right)$ steps.

\section{The Weak Shared Coin Protocol}

In this section we show how to transform our exponential protocol into a polynomial protocol in which processes achieve agreement after an expected $O\left(n^{2}\right)$ writes and $O\left(n^{4}\right)$ reads. The basic idea is to replace the independent coin flips with a weak shared coin object that, in essence, simulates a coin shared by all processes. The weak shared coin protocol is parameterized by a value $K>1$. It has the key property that any adversary scheduler has only a weak influence over the protocol's outcome:

No adversary can ensure that some process will observe a particular outcome (e.g., heads) with probability exceeding $(\boldsymbol{K}+1) / 2 \boldsymbol{K}$.

The bias that can be introduced by any adversary scheduler is thus independent of $n$, and asymptotically approaches zero as $K$ increases. We remark that the protocol does not guarantee that all processes observe the same outcome, but Property 1 implies that they do so with probability greater than $(K+1) / 2 K$.

We make the following modification to the consensus protocol. The processes share an unbounded array of weak shared coins. At round $r$, instead of flipping independent coins, the processes collectively flip the $r^{\text {th }}$ weak shared coin.

Theorem 10: The revised protocol terminates in $O(1)$ rounds.

Proof: A process is deterministic at round $r$ if it does not flip a coin at that round. If there are no deterministic processes, the protocol terminates if all non-deterministic processes randomly choose the same new preference (Lemma 4). Otherwise, Lemma 7 implies that all deterministic processes at round $r$ have identical preferences at that round, say $v$, and that the protocol terminates if all non-deterministic processes randomly choose preference $v$ (Lemma 4). In either case. Property 1 ensures that the protocol terminates at round $r$ with likelihood at least $(K-1) / 2 K$. The protocol is thus a Bemoulli process with expected running time less than or equal to $2 K /(K-1)$, independent of $n$.

The complexity of achieving consensus in terms of primitive reads and writes is thus the complexity of implementing the weak shared coin. 


\subsection{Implementing a Weak Shared Coin}

The weak shared coin protocol is implemented using a shared counter abstraction, whose implementation in terms of reads and writes is given below. The counter is initially zero, and it provides the following operations:

inc $=\operatorname{proc}(c:$ counter $)$

increments the counter,

dec $=$ proc $(c:$ counter $)$

decrements the counter, and

readCounter $=$ proc $(c:$ counter) returns (int)

returns the counter's current value.

The protocol is shown in in Figure 5-1. The processes collectively undertake a random walk: each process flips an unbiased coin, and depending on the outcome, increments or decrements the shared counter. It then reads the counter. If the observed value is greater than or equal to $K n$, the process decides heads, and if the observed value is less than or equal to $-K n$, it decides tails. Informally, the only way the adversary can influence the outcome of the protocol is to suspend processes that are about to move the counter in the undesired direction. After suspending $n-1$ such processes, however, the adversary has "used up" its influence, and the remaining process is free to wander at random. As $K$ increases, the importance of this bias decreases.

Let $H$ and $T$ be the respective number of heads and tails generated so far.

Lemma 11: If $H-T<-(K+1) n$ then all undecided processes will eventually decide tails.

Proof: Since the adversary can suspend at most one write per process, the counter value read by any process can differ from $H-T$ by at most $n-1$. Once $H-T$ falls below $-(K+1) n$, every process that samples the counter will observe a value less than or equal to $K n$.

By similar reasoning:

Lemma 12: If some process decides heads, then at the time of its last read, $H-T \geq(K-1) n$.

We can combine these two observations to derive a bound on the likelihood the adversary can force disagreement, or a desired outcome.

Theorem 13: The adversary can force processes to disagree with probability less than or equal to $(K-1) / 2 K$.

Theorem 14: The adversary can force some process to flip heads with probability less than or equal to $(K+1) / 2 K$.

Proof: Lemma 12 implies that no process can decide heads before $H-T$ reaches $(K-1) n$ for the first time. If, however, $H-T$ falls below $-(K+1) n$ before reaching $(K-1) n$, then Lemma 11 implies that no process can decide heads. If we make the conservative assumption that the adversary can force some undecided process to choose heads if $H-T \geq(K-1) n$, then the value of $H-T$ can be viewed as a random walk starting at the origin with absorbing barriers at $-(K+1) n$ (all decide tails) and $(K-1) n$ (some may decide heads). It is a standard result of random walk theory [18;Ch.XIV] that the probability of reaching $(K-1) n$ before $-(K+1) n$ is $(K+1) / 2 K$.

Theorem 15: The expected running time of the protocol is $O\left(n^{2}\right)$ rounds.

Proof: Instead of promoting a particular outcome, suppose the adversary adopts a dilatory strategy, seeking to prolong the protocol for as long as possible. As noted above, the protocol will terminate whenever the absolute value of the counter exceeds $(K+1) n$, thus the protocol behaves like a random walk staring at the origin with absorbing barriers at $(K+1) n$ and $-(K+1) n$. It is a standard result of random walk theory [18] that the expected running time of such a walk is $(K+1)^{2} n^{2}$, i.e. $O\left(n^{2}\right)$. 


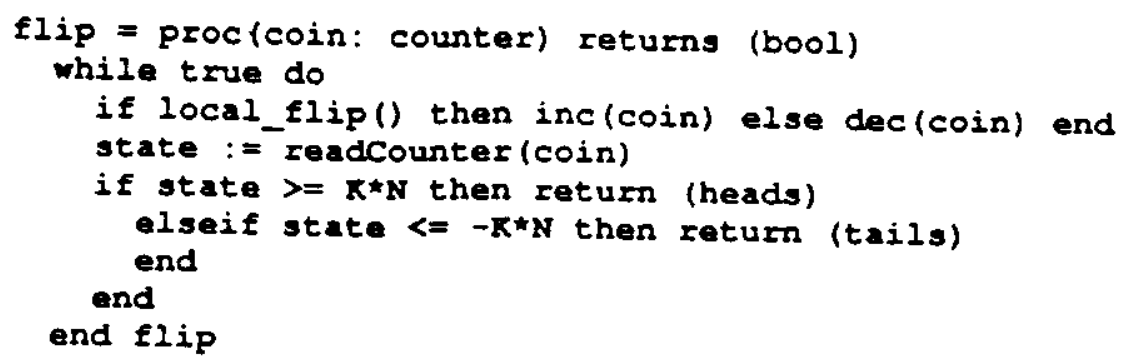

Figure 5-1: The Weak Shared Coin Protocol

\section{The Counter Abstraction}

The counter implementation is a straightforward adaptation of an algorithm proposed by Lamport [24] for $\mathrm{read} / \mathrm{write}$ registers. The counter is represented by an $n$-element array of registers, one for each process. Each register has two fields: a count field incremented whenever that process alters the register's value, and a val field representing that process's contribution to the current counter value. To increment or decrement the counter, $P$ overwrites its register with a new value whose count field is incremented, and whose val field is incremented or decremented. To read the counter it scans the array twice: if both scans yield identical values, the read returns the sum of the val fields, otherwise the read is restarted.

Note that the inc and dec operations are wait-free, but readCounter can be starved if it is internupted by an infinite sequence of writers. The adversary cannot exploit this property to force the protocol to run forever: after enough writes, the next reader will drop out of the protocol, and because there are only finitely many processes, it will eventually be possible for some process to complete a read.

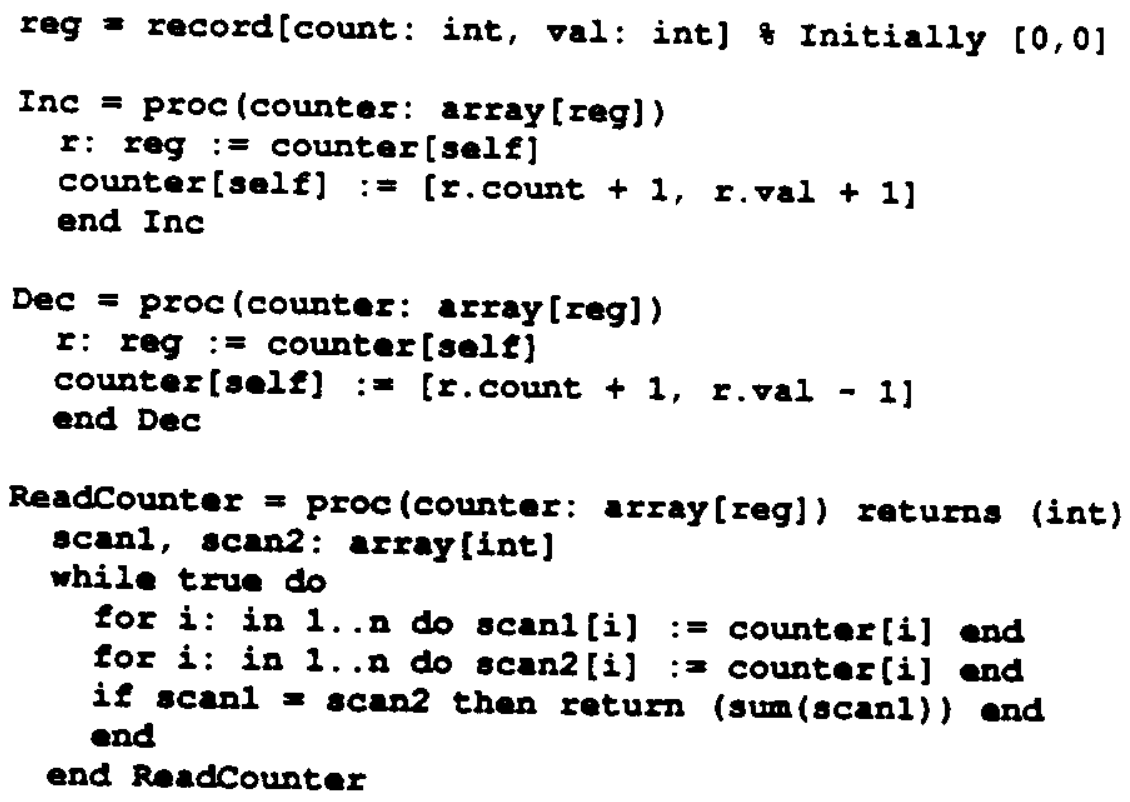

Figure 6-1: Implementation of Shared Counter

Each inc or dec translates into to a single write, so the $O\left(n^{2}\right)$ expected steps needed to exhaust the random walk translate into an expected $O\left(n^{2}\right)$ primitive write operations. Each write can disnupt readCounter operations by $n-1$ processes, and each of these must then undertake $n$ additional primitive reads, thus the $O\left(n^{2}\right)$ expected steps result in an expected $O\left(n^{4}\right)$ primitive read operations. 


\section{Consensus Using Fetch-And-Add}

The fetch-and-add operation [23] atomically adds a quantity to a register and returns the register's old value. Fetch-and-add solves consensus deterministically for two processes, but not for three or more [21]. Figure 7-1 shows a weak shared coin implementation using fetch-and-add operations. Not surprisingly, fetch-and-add is more efficient than read and write; it is straightforward to show that this protocol completes in an expected $\mathrm{O}\left(n^{2}\right)$ total operations.

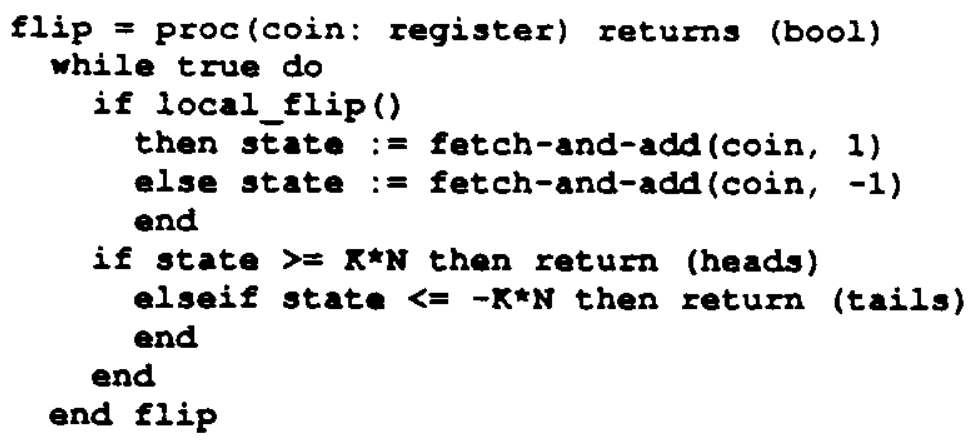

Figure 7-1: The Weak Shared Coin Protocol using Fetch-And-Add

\section{Strong Shared Coin Protocols}

A strong shared coin protocol is a consistent wait-free algorithm by which $n$ processes agree on a value in (heads, tails) by applying operations to a shared object. A shared coin protocol is unbiased if both choices are equally likely; i.e., the adversary has no control over the outcome. A naive solution might have each process flip an unbiased local coin to choose its input value, and then achieve consensus with the others. Such a solution is heavily biased, however, since an adversary that "wants" an outcome of heads will run only the processes that prefer heads. Against such an adversary, this naive protocol will decide tails only if all processes initially flip tails, a probability of $1 / 2^{n}$.

Theorem 16: An unbiased strong shared coin protocol is impossible.

Proof: By contradiction. For any two-process protocol, we construct an adversary that produces heads with probability greater than $1 / 2$. Assume we have an unbiased protocol, and let $P$ and $Q$ be the two processes. Define a configuration's bias to be the maximum probability of eventually deciding heads from that configuration, where the maximum is taken over all possible adversaries. Define a process's current preference to be the probability it will eventually decide heads if it is run uninternuptedly until it decides. For an unbiased protocol, the initial configuration's bias is $1 / 2$, as is each process's preference.

Consider the following adversary. Run $P$ until it is about to take a step that changes the current bias. Such a step must eventuaily occur, because the protocol cannot nun forever. Moreover, that step must be a coin flip internal to $\mathrm{P}$, since all other steps are deterministic and under the adversary's control. Before the coin flip, the configuration's bias is 1/2, as are P and Q's preferences. Suppose P's local flip yields heads with probability $h$. Let $b_{h}\left(b_{l}\right)$ be the bias resulting if $P$ flip heads (tails). Since the protocol is unbiased, we have:

$$
1 / 2=h \cdot b_{h}+(1-h) \cdot b_{l},
$$

implying that one resulting bias is greater than $1 / 2$ and one less. Assume $b_{h}>1 / 2>b_{t}$; the other case is symmetric. Since $Q$ cannot directly observe $P$ 's coin flip, its preference continues to be $1 / 2$.

If the outcome is heads, the definition of bias implies that the adversary can ensure an outcome of heads with probability $b_{h}$. If the outcome is tails, the adversary runs $Q$ uninterruptedly until it decides, ensuring an outcome of heads with probability $1 / 2$. Taken together, the adversary can ensure heads with probability:

$$
h \cdot b_{h}+(1-h) / 2 \text {. }
$$



Since $b_{h}>1 / 2$, however, this quantity exceeds $1 / 2$, contradicting the hypothesis that the protocol is
unbiased.

Note that this proof makes no assumptions about how processes communicate; they could use read/write registers, fetch-and-add registers, messages, or other objects.

Although we have shown that the adversary can always introduce some bias, we have given no indication of how large that bias must be. A shared coin protocol is asymptotically unbiased if the bias introduced by the adversary can be made arbitrarily small.

Theorem 17: A asymptotically unbiased strong shared coin protocol with expected running ume polynomial in the number of processes is possible using shared read/write registers.

Proof: Have each process choose heads or tails using a weak shared coin, and then run the polynomial consensus protocol given above. The adversary can influence the outcome by biasing the initial preferences. If any process prefers heads, the adversary can suspend the others, while if all processes prefer tails, the adversary has no more control. The likelihood the adversary can force some process to choose heads in the initial round is thus $(K+1) / 2 K$, which approaches $1 / 2$ as $K$ increases.

\section{Discussion}

Most recent work on wait-free synchronization has focused on the construction of atomic read/write registers $[5,9,24,25,29,30,31,33]$. Starting with "safe" bits for which overlapping read and write operations have unpredictable effects, these papers describe a sequence of algorithms for constructing wait-free implementations of $\mathrm{read} / \mathrm{write}$ registers providing successively stronget guarantees, culminating in algorithms that permit multiple concurrent readers and writers, an impressive achievement.

Nevertheless, reading and writing to individual registers is not the level of abstraction at which most programs are written. Wait-free synchronization will be useful in practice only if it is possible to construct wait-free implementations of objects with richer semantics than registers, objects such as test-and-set registers, stacks, queues, file system directories, databases, etc. It is known, however, that atomic read/write registers have few, if any, interesting applications in this area [21]. Using atomic read/write registers, it is impossible to construct a wait-free implementation of: (1) common data types such as sets, queues, stacks, priority queues, or lists, (2) most if not all the classical synchronization primitives such as test-and-set, compare-and-swap, and fetch-and-add, and (3) such simple memory-to-memory operations as move or memory-to-memory swap.

One way to interpret these impossibility results is that atomic read/write registers are a computational dead-end, and that wait-free synchronization is unrealizable by machine architectures in which processes communicate by reading and writing shared memory locations. The results in this paper suggest an alternative position. If one can achieve consensus, one can transform a sequential implementation of any object whose operations are total (i.e., defined in every state) to a wait-free linearizable implementation [21], where each operation requires at most $n$ rounds of consensus. In the same way, the randomized consensus protocol presented here can be used to transform any sequential object implementation into a randomized wait-free implementation, where each operation has expected running time polynomial in the number of processes. In short, wait-free synchronization is indeed realizable under conventional architectures, provided the wait-free guarantee is probabilistic in nature.

\section{Acknowledgment}

The authors would like to thank Hagit Attiya for her comments. 


\section{References}

[1] K. Abrahamson.

On achieving consensus using a shared memory.

In Seventh ACM SIGACT-SIGOPS Symposium on Principles of Distributed Computing. August, 1988.

[2] J.H. Anderson and M.G. Gouda.

The Virtue of Patience: Concurrent Programming With and Without Waiting.

Private Communication.

[3] M. Ben-Or.

Another advantage of free choice: completely asynchronous agreement protocols.

In Second ACM SIGACT-SIGOPS Symposium on Principles of Distributed Computing, pages 27-30.

August, 1983.

[4] M. Ben-Or and N. Linial.

Collective coin flipping, robust voting schemes, and minima of Banzhaf values.

In Twenty-sixth Annual Symposium on Foundations of Computer Science, pages 408-416. October, 1985.

[5] B. Bloom.

Constructing two-writer atomic registers.

In Proceedings of the Sixth ACM Symposium on Principles of Distributed Computing, pages 249-259. 1987.

[6] G. Bracha and S. Toueg.

Resilient Consensus Protocols.

In Second ACM SIGACT-SIGOPS Symposium on Principles of Distributed Computing, pages 12-26.

August, 1983.

[7] G. Bracha.

An $\mathrm{O}(\log n)$ expected rounds randomized byzantine generals algorithm.

In Seventeenth Annual Symposium on Theory of Computation. 1985.

[8] A. Broder and D. Dolev.

Flipping coins in many pockets (byzantine agreement on uniformly random values.

In Twenty-Fifth Annual Symposium on Foundations of Computer Science, pages 157-170. October, 1984.

[9] J.E. Bums and G.L. Peterson.

Constructing Multi-reader atomic values from non-atomic values.

In Proceedings of the Sixth ACM Symposium on Principles of Distributed Computing, pages 222-231. 1987.

[10] B. Chor, M. Merritt, and D.B. Shmoys.

Simple constant-time consensus protocols in realistic failure models.

In Proceedings of the Fourth ACM Symposium on Principles of Distributed Computing, pages 152-160. 1985.

[11] B. Chor and B. Coan.

A simple and efficient randomized byzantine agreement algorithm.

IEEE Transactions on Sofware Engineering SE-11(6):531-539, June, 1985.

[12] B. Chor, A. Israeli, and M. Li.

On processor coontination using asynchronous hardware.

In Proceedings of the Sixth ACM Symposium on Principles of Distributed Computing, pages 86-97. 1987.

[13] B. Coan and J. Lundelius.

Transaction Commit in a Realistic Fault Model.

In Fifth ACM SIGACT-SIGOPS Symposium on Principles of Distributed Computing, pages 40-52. August, 1986.

[14] D. Dolev, C. Dwork, and L Stockmeyer.

On the minimal synchronism needed for distributed consensus .

Journal of the ACM 34(1):77-97, January, 1987. 
[15] C. Dwork, D. Shmoys, and L. Stockmeyer.

Flipping persuasively in constant expected time.

In Twenty-Seventh Annual Symposium on Foundations of Computer Science, pages 222-232. October, 1986.

[16] C. Dwork, N. ..., nch, and L Stockmeyer.

Consensus in the Presence of Partial Synchrony .

Journal of the ACM 35(2):228-323, April, 1988.

[17] P. Feldman and S. Micali.

Optimal Algorithms for Byzantine Agreement.

In Twentieth Annual ACM Symposium on Theory of Computing, pages 148-161. May, 1988.

[18] W. Feller.

An Introduction to Probability Theory and its Applications.

John Wiley \& Sons, 1957.

[19] M. Fischer, N.A. Lynch, and M.S. Paterson.

Impossibility of distributed commit with one faulty process.

Journal of the ACM 32(2), April, 1985.

[20] M.P. Herlihy and J.M. Wing.

Axioms for concurrent objects.

In 14th ACM Symposium on Principles of Programming Languages, pages 13-26. January, 1987.

[21] M.P. Herlihy.

Impossibility and Universality Results for Wait-Free Synchronization.

In Seventh ACM SIGACT-SIGOPS Symposium on Principles of Distributed Computing. August, 1988.

[22] J.G. Kemeny, J.L. Snell, and A.W. Kapp.

Denumerable Markov Chains.

D. Van Nostrand, 1966.

[23] C.P. Kruskal, L. Rudolph, and M. Snir.

Efficient Synchronization on Multiprocessors with Shared Memory.

In Fifth ACM SIGACT-SIGOPS Symposium on Principles of Distributed Computing. August, 1986.

[24] L. Lamport.

Concurrent Reading and Writing.

Communications of the ACM 20(11):806-811, November, 1977.

[25] L. Lamport.

On Interprocess Communication, Parts I and II.

Distributed Computing 1:77-101, 1986.

[26] M.C. Loui and H.H. Abu-Amara.

Memory Requirements for Agreement Among Unreliable Asynchronous Processes.

Advances in Computing Research.

JAI Press, 1987, pages 163-183.

[27] N.A. Lynch and M. Merritt.

Introduction to the Theory of Nested Transactions.

Technical Report MIT/LCS/TR-387, Massachusetts Institute of Technology Laboratory for Computer Science, April, 1986.

[28] N.A. Lynch and M.R. Tuttle.

Hierarchical Correctness Proofs for Distributed Algorithms.

Technical Report MIT/LCS/TR-387, Massachusetts Institute of Technology Laboratory for Computer Science, April, 1987.

[29] R. Newman-Wolfe.

A Protocol for wait-free, atomic, multi-reader shared variables.

In Proceedings of the Sixth ACM Symposium on Principles of Distributed Computing, pages 232-249. 1987. 
[30] GL. Peterson.

Concurrent reading while writing.

ACM Transactions on Programming Languages and Systems 5(1):46-55, January, 1983.

[31] G.L. Peterson and J.E. Burns.

Concurrent reading while writing II: the multi-writer case.

Technical Report GIT-ICS-86/26, Georgia Institute of Technology, December, 1986.

[32] M. Rabin.

Randomized Byzantine Generals.

In Twenty-fourth Annual Symposium on Foundations of Computer Science, pages 403-409. October, 1983.

[33] A.K. Singh, J.H. Anderson, and M.G. Gouda.

The elusive atomic register revisited.

In Proceedings of the Sixth ACM Symposium on Principles of Distributed Computing, pages 206-221. August, 1987. 